J. Igusa

Nagoya Math. J.

Vol. 47 (1972), 169-198

\title{
ON THE ARITHMETIC OF PFAFFIANS ${ }^{1}$
}

\author{
To Chow on his 60th Birthday
}

\author{
JUN-ICHI IGUSA
}

In this paper, we shall supply proofs to the results announced in [2], pp. 74-75: we shall prove the Siegel formula for the Pfaffian of degree $n$ over an algebraic number field and also determine the zeta function of the Pfaffian. In the appendix, we shall briefly discuss the non-split case where the Pfaffian is replaced by the norm form of the simple Jordan algebra of quaternionic hermitian matrices of degree $n$.

The arithmetic of Pfaffians is a very special topic; yet it has the "generality" of being a typical example of the arithmetic of invariants of "absolutely admissible representations". We can state a conjectural Siegel formula for any absolutely admissible representation. However, at the present moment, the only approach to this conjecture is by a caseby-case examination via the classification of such representations [cf. 3]. And we shall settle one of the cases in this paper.

We recall that Siegel's works on quadratic forms [cf. 6] were recast by Weil in the framework of adele geometry [cf. 7, 8,9]; also a similar problem was discussed by Mars [5] for the norm form of the simple exceptional Jordan algebra. We shall use their results and methods especially in the later parts of this paper.

1. Pfaffians. Let $\Omega$ denote a "universal domain" of arbitrary characteristic and $V$ a vector space over $\Omega$ of dimension $m$; consider the exterior algebra $E(V)$ of $V$. Then $E(V)$ is a non-commutative graded ring over $E(V)_{0}=\Omega$ satisfying

$$
\operatorname{dim} E(V)_{p}=\left(\begin{array}{c}
m \\
p
\end{array}\right)(0 \leqq p \leqq m), \quad E(V)_{p}=0(p>m)
$$

moreover, for every $x$ in $E(V)_{p}$ and $y$ in $E(V)_{q}$, we have $x y=(-1)^{p q} \cdot y x$. Therefore

Received December 23, 1971.

1 This work was partially supported by the National Science Foundation. 


$$
A=\sum_{p \geqq 0} E(V)_{2 p}
$$

is an Artinian local ring with $A_{+}=E(V)_{2}+E(V)_{4}+\cdots$ as its maximal ideal. According to Chevalley [1], there exists a unique homomorphism "exp" of the additive group of $A_{+}$to the unit group of $A$ with the property that, for every $v_{1}, \cdots, v_{2 p}$ in $V, \exp \left(v_{1} \cdots v_{2 p}\right)=1+v_{1} \cdots v_{2 p}$.

We observe that $G L(V)$ acts on $E(V)$ as a group of degree-preserving automorphisms. Since $E(V)_{m}$ is of dimension 1 over $\Omega, G L\left(E(V)_{m}\right)$ is canonically isomorphic to $\Omega^{\times}$. Putting these together, we get an intrinsically defined homomorphism "det" of $G L(V)$ to $\Omega^{\times}$.

Suppose that $m=2 n$ and take an $\Omega$-base $e_{1}, \cdots, e_{2 n}$ of $V$; we identify an element $x$ of $E(V)_{2}$ with the alternating matrix of degree $2 n$ with the $x_{i j}$ defined by

$$
x=\sum_{i<j} x_{i j} e_{i} e_{j}
$$

as its $(i, j)$-coefficient. Then the Pfaffian $P f(x)$ of $x$ is given by

$$
\exp (x)=1+\cdots+\operatorname{Pf}(x) e_{1} \cdots e_{2 n} .
$$

We identify an element $g$ of $G L(V)$ with the element of $G L_{2 n}(\Omega)$ with the $g_{i j}$ defined by

$$
g \cdot e_{j}=\sum_{i=1}^{2 n} e_{i} g_{i j} \quad(1 \leqq j \leqq 2 n)
$$

as its $(i, j)$-coefficient. Then $\operatorname{det}(g)$ becomes the determinant of the matrix $g$; moreover, $\exp (g \cdot x)=g \cdot \exp (x)$ implies that

$$
P f\left(g x^{t} g\right)=\operatorname{det}(g) P f(x) .
$$

The proof of the following properties of $P f$ is equally formal and straightforward:

LEMMA 1. Let $x$ denote an alternating matrix of degree $2 n$ and $X_{i j}$ the submatrix of $x$ which is obtained from $x$ by crossing out its $i$-th and $j$-th rows and columns; put

$$
\xi_{i j}=(-1)^{i+j} \operatorname{P} f\left(X_{i j}\right)
$$

for $i<j$ and denote by $\xi$ the alternating matrix of degree $2 n$ with $\xi_{i j}$ as its $(i, j)$-coefficient; then we have

$$
x \xi=\xi x=P f(x) 1_{2 n} .
$$


Moreover, if we put $\alpha=x_{2 n-1,2 n}, y=x_{2 n-1,2 n}, u_{i}=x_{i, 2 n-1}, v_{i}=x_{i, 2 n}$ for $1 \leqq$ $i \leqq 2 n-2$, and if we denote by $\eta$ the matrix " $\xi$ " for $y$, we have

$$
P f(x)=\alpha \cdot P f(y)+u \eta^{t} v
$$

if we regard $u \eta^{t} v$ as a quadratic form $Q_{y}(z)$ in $z=(u, v)$, the discriminant of $Q_{y}(z)$ is equal to $\operatorname{det}(\eta)^{2}$.

We recall that if we have a quadratic form $Q(z)=\frac{1}{2} z S^{t} z$, in which $S={ }^{t} S$, $\operatorname{det}(S)$ is called the discriminant of $Q(z)$. The well-known property of $P f: \operatorname{det}(x)=P f(x)^{2}$, which is used in the usual definition of $P f(x)$, does not seem to come out formally from the above setup; it follows from some observations in the next section. At any rate, if we use that property of $P f$, we have $\operatorname{det}(\xi)=\operatorname{det}(x)^{n-1}$ and $\operatorname{det}(\eta)=\operatorname{det}(y)^{n-2}$.

2. Orbital structure. Let $m$ denote a positive integer and put $G^{*}=$ $G L_{m}\left(=G L_{m}(\Omega)\right), G=S L_{m}$. If $X$ denotes the vector space of alternating matrices of degree $m$, the group $G^{*}$, hence also $G$, acts on $X$ as $x \rightarrow$ $g x^{t} g$. If we put

$$
\rho(g) \cdot x=g x^{t} g,
$$

we get a rational representation $\rho$ of $G^{*}$, hence also of $G$, in $X$; all these are "defined" over an arbitrary subfield $k$ of $\Omega$. For the sake of completeness, we shall review the classical description of $G$-orbits in $X$.

Suppose first that $m$ is odd. Then, if we denote by $U_{r}$ the set of points of $X$ of rank $2 r$ in which $0 \leqq r<\frac{1}{2} m$, each $U_{r}$ forms a $G$-orbit. More precisely, if we denote by $e_{r}$ the "standard alternating matrix" of degree $2 r$, i.e., if we put

$$
e_{r}=\left[\begin{array}{rl}
0 & 1_{r} \\
-1_{r} & 0
\end{array}\right]
$$

then, for every $x$ in $U_{r}$ which is rational over a field $K$, there exists an element $g$ of $G_{K}$ satisfying

$$
\rho(g) \cdot x=\left[\begin{array}{ll}
e_{r} & 0 \\
0 & 0
\end{array}\right] .
$$

In particular, each $U_{r}$ satisfies the Witt condition relative to any field $k$. (Suppose that $U$ is the quotient variety, defined over a field $k$, of an algebraic group $G$ by a Zariski closed subgroup of $G$; then, the "Witt 
condition" relative to $k$ states that, for any extension field $K$ of $k, G_{K}$ acts transitively on $U_{K}$.) We put $U(0)=U_{\frac{1}{2}(m-1)}$.

Suppose next that $m$ is even; put $m=2 n$. We shall denote by $U_{r}$ the set of points of $X$ of rank $2 r$ in which $0 \leqq r<n$. Then each $U_{r}$ forms a $G$-orbit; in fact, we have

$$
U_{r}=\rho(G) \cdot\left[\begin{array}{ll}
e_{r} & 0 \\
0 & 0
\end{array}\right]
$$

For every $i$ in $\Omega$, let $d$ denote the diagonal matrix of degree $n$ with $1, \cdots, 1,(-1)^{\frac{1}{2} n(n-1)} i$ as its diagonal coefficients; put

$$
U(i)=\rho(G) \cdot\left[\begin{array}{rr}
0 & d \\
-d & 0
\end{array}\right]
$$

Then we have $U_{n-1}=U(0)$. Moreover $U_{0}, U_{1}, \cdots, U_{n-2}$ and $U(i)$ for all $i$ in $\Omega$ form the complete set of $G$-orbits in $X$; each $G$-orbit in $X$ satisfies the Witt condition relative to any field $k$.

Since we have $\operatorname{Pf}(\rho(g) \cdot x)=\operatorname{det}(g) P f(x)$ for every $g$ in $G^{*}$ and $x$ in $X, P f$ is a $G$-invariant on $X$. We recall that $P f(x)$ is a homogeneous polynomial of degree $n$ in $x_{i j}(i<j)$ with \pm 1 as its coefficients. Moreover, if $d$ is as above, the value of $P f$ at the alternating matrix of degree $2 n$ composed of $0, d,-d, 0$ is precisely $i$. Therefore, if we denote the morphism $X \rightarrow \Omega$ defined by $x \rightarrow P f(x)$ also by $P f$, we have $(P f)^{-1}(i)=$ $U(i)$ for every $i \neq 0$ and

$$
(P f)^{-1}(0)=\left(\coprod_{i=0}^{n-2} U_{i}\right) \amalg U(0) .
$$

We shall show that $P f$ is the only $G$-invariant on $X$, i.e., every $G$ invariant on $X$ is a polynomial in $P f$. Let $f$ denote a $G$-invariant on $X$ defined over a field $K$, i.e., such that all coefficients of $f$ are in $K$; let $x$ denote a generic point of $X$ over $K$. Then we have

$$
f(x)=f\left[\begin{array}{rr}
0 & d \\
-d & 0
\end{array}\right]
$$

in which $d$ is the diagonal matrix with $1, \cdots, 1,(-1)^{\frac{1}{2} n(n-1)} P f(x)$ as its diagonal coefficients. We observe that $P f(x)$ is transcendental over $K$ and that $f(x)$ is expressed as a polynomial in $P f(x)$ with coefficients in $K$. This proves the assertion. 
LEMMA 2. Let $x$ denote an arbitrary point of $X$; then $x$ is simple on the hypersurface in $X$ defined by $P f-P f(x)=0$ if and only if $\operatorname{rank}(x) \geqq 2 n-2$.

Proof. For our purpose, we can replace $x$ by any other point of $\rho(G) \cdot x$. Therefore we shall replace $x$ by

$$
x_{0}=\left[\begin{array}{rr}
0 & d \\
-d & 0
\end{array}\right] \text {, }
$$

in which $d$ is a diagonal matrix with $d_{1}, \cdots, d_{n}$ as its diagonal coefficients. If we denote by $\delta$ the "differentiation", we have

$$
(\delta P f)\left(x_{0}\right)= \pm \sum_{i=1}^{n}\left(d_{1} \cdots d_{i-1} d_{i+1} \cdots d_{n}\right) \delta x_{i, n+i} .
$$

Hence $(\delta P f)\left(x_{0}\right) \neq 0$ if and only if at most one of the $d_{1}, \cdots, d_{n}$ is 0 , i.e., if and only if $\operatorname{rank}\left(x_{0}\right) \geqq 2 n-2$. We observe that $(\delta P f)\left(x_{0}\right) \neq 0$ is the "Jacobian criterion" for $x_{0}$ to be simple on the hypersurface in $X$ defined by $P f-P f\left(x_{0}\right)=0$. q.e.d.

In the following lemma, $m$ can be even or odd:

LemMA 3. Let $x$ denote a rational point of $X$ over a field $K$ and $H$ the stabilizer of $G$ at $x$; then $H$ is a connected, simply connected algebraic group defined over $K$. Moreover, if $\operatorname{rank}(x)=2 r, H$ is isomorphic over $K$ to the following semidirect product:

$$
\left(S p_{2 r} \times S L_{m-2 r}\right) \cdot\left(G_{a}\right)^{2 r(m-2 r)} .
$$

Proof. For our purpose, we can replace $x$ by any other point of $\rho\left(G_{K}\right) \cdot x$. Therefore, if $r=\frac{1}{2} m$, we shall replace $x$ by

$$
x_{0}=\left[\begin{array}{rr}
0 & d \\
-d & 0
\end{array}\right] \text {, }
$$

in which $d$ is the diagonal matrix with $1, \cdots, 1,(-1)^{\frac{1}{2} n(n-1)} P f(x)$ as its diagonal coefficients; then we have

$$
H=\left[\begin{array}{ll}
d & 0 \\
0 & 1_{r}
\end{array}\right] S p_{2 r}\left[\begin{array}{ll}
d & 0 \\
0 & 1_{r}
\end{array}\right]^{-1}
$$

If $r<\frac{1}{2} m$, we shall replace $x$ by

$$
x_{0}=\left[\begin{array}{ll}
e_{r} & 0 \\
0 & 0
\end{array}\right]
$$


write an arbitrary element $g$ of $G$ as

$$
g=\left[\begin{array}{ll}
u_{1} & u_{12} \\
u_{21} & u_{2}
\end{array}\right]
$$

in which $u_{12}$ is a rectangular matrix with $2 r$ rows and $m-2 r$ columns. Then we have $\rho(g) \cdot x_{0}=x_{0}$ if and only if $u_{1}$ is in $S p_{2 r}$ and $u_{21}=0$. Since $\operatorname{det}(g)=\operatorname{det}\left(u_{1}\right)=1, u_{2}$ is in $S L_{m-2 r}$; also, if we write

$$
g=\left[\begin{array}{ll}
u_{1} & 0 \\
0 & u_{2}
\end{array}\right]\left[\begin{array}{ll}
1_{2 r} & v_{12} \\
0 & 1_{m-2 r}
\end{array}\right]
$$

the second factors form a normal subgroup of $H$, which is isomorphic to the additive group of $v_{12}$. q.e.d.

If $x$ is as in Lemma 3 , we have

$$
\operatorname{dim}(G / H)=r(2 m-2 r-1)
$$

for $r<\frac{1}{2} m$; in the case where $m$ is even and $r=\frac{1}{2} m$, this has to be modified as $\operatorname{dim}(G / H)=r(2 m-2 r-1)-1$. Therefore we have $\operatorname{dim} U(0)$ $-\operatorname{dim}(X-U(0))=3$ for every odd $m \geqq 3$ and

$$
\operatorname{dim} U(0)-\operatorname{dim}\left((P f)^{-1}(0)-U(0)\right)=5
$$

for every even $m \geqq 4$. We put $X^{\prime}=U(0)$ if $m$ is odd and

$$
X^{\prime}=\coprod_{i \in \Omega} U(i)
$$

if $m$ is even, and we call $X^{\prime}$ the principal subset of $X$. We shall denote by $\theta_{i}$ the residue (in the sense of algebraic geometry) of $(P f(x)-i)^{-1} d x$ on $U(i)$, in which $d x=\wedge_{j<k} d x_{j k}$; this is a $G$-invariant gauge form on $U(i)$.

3. Elementary arithmetric. Let $o$ denote a discrete valuation ring and $\boldsymbol{p}$ the ideal of non-units of $\boldsymbol{o}$ such that $\boldsymbol{o} / \boldsymbol{p}$ is finite; let $q$ denote the number of elements in $\boldsymbol{o} / \boldsymbol{p}$. Let $X^{0}$ denote the $\boldsymbol{o}$-module of alternating matrices of degree $2 n$ with coefficients in $o$. For every positive integer $m$ and every element $i$ of $o$, we shall calculate the number, say $N$, of cosets in $X^{0} / p^{m} X^{0}$ defined by the following congruence:

$$
P f(x) \equiv i \bmod p^{m} \text {. }
$$

We observe that the correspondence $g \rightarrow \operatorname{det}(g)$ defines a surjective homomorphism of $G L_{2 n}(\boldsymbol{o})$ to the group of units of $\boldsymbol{o}$. Since $G L_{2 n}(\boldsymbol{o})$ acts 
on $X^{0}$, and also on $X^{0} / p^{m} X^{0}$, as $x \rightarrow g x^{t} g$, by using the formula $P f\left(g x^{t} g\right)$ $=\operatorname{det}(g) P f(x)$, we see that $N$ depends only on the order of $i$. If $e$ is the order of $i$, i.e., if $\boldsymbol{p}^{e}=\boldsymbol{o} i$ for $i \neq 0$ and $e=\infty$ for $i=0$, we put $N=\dot{F}_{n}(m, e)$. Since we have $\boldsymbol{F}_{n}(m, e)=\boldsymbol{F}_{n}(m, m)$ for every $e \geqq m$, we may assume that $e \leqq m$. We shall denote by $f_{n}(m, e)$ the quotient of $\boldsymbol{F}_{n}(m, e)$ by the total number of cosets in $X^{0} / \boldsymbol{p}^{m} X^{0}$, i.e., we put

$$
f_{n}(m, e)=F_{n}(m, e) / q^{m n(2 n-1)} .
$$

We shall compute $f_{n}(m, e)$ explicitly:

LEMMA 4. In the case where $e<m$, we have

$$
f_{n}(m, e)=q^{-m} \prod_{i=1}^{n-1}\left(1-q^{-(2 i+1)}\right)\left(1-q^{-2 i}\right)^{-1}\left(1-q^{-2(i+e)}\right)
$$

with the understanding that the product represents 1 for $n=1$.

Proof. Since we have $P f(x)=x_{12}$ for $n=1$, we get $F_{1}(m, e)=1$, hence $f_{1}(m, e)=q^{-m}$ for every $e$. This coincides with the proposed expression for $f_{n}(m, e)$ in the case where $n=1$. We shall obtain a recursion formula for $f_{n}(m, e)$ with respect to $n$ assuming that $n \geqq 2$. By Lemma 1 , we have

$$
P f(x)=\sum_{i=1}^{2 n-1}(-1)^{i-1} P f\left(x_{i}\right) y_{i}\left(y_{i}=x_{i, 2 n}\right),
$$

in which $x_{i}$ is the submatrix of $x$ obtained from $x$ by crossing out its $i$-th and $2 n$-th rows and columns. We shall denote by $y$ the column vector with $y_{1}, \cdots, y_{2 n-1}$ as its coefficients. Let $\pi$ denote an element of order 1, i.e., an element of $\boldsymbol{p}-\boldsymbol{p}^{2}$, and $\pi^{j}$ the greatest common divisor of the coefficients of $y$. If $x$ satisfies the congruence $P f(x) \equiv \pi^{e} \bmod p^{m}$ for $e<m$, we necessarily have $j \leqq e$. Consider the set of column vectors $y \bmod \boldsymbol{p}^{m}$ such that the greatest common divisor of the coefficients of each $y$ is $\pi^{j}$. The number of elements in this set is clearly equal to $\left(q^{m-j}\right)^{2 n-1}$ $\left(1-q^{-(2 n-1)}\right)$. Moreover $S L_{2 n-1}(\boldsymbol{o})$ acts transitively on the set as $y \rightarrow u^{\prime} y$. We observe also that $S L_{2 n-1}(\boldsymbol{o})$ acts, as

$$
x \rightarrow\left[\begin{array}{ll}
u^{\prime} & 0 \\
0 & 1
\end{array}\right] x^{t}\left[\begin{array}{cc}
u^{\prime} & 0 \\
0 & 1
\end{array}\right],
$$

on $X^{0} / \boldsymbol{p}^{m} X^{0}$ and on its subset defined by $P f(x) \equiv \pi^{e} \bmod \boldsymbol{p}^{m}$ and by the additional condition that the greatest common divisor of the coefficients 
of $y$ is $\pi^{j}$. Consequently, the number of elements in this subset is equal to the product of the above number of $y \bmod \boldsymbol{p}^{m}$ and the number of $x$ $\bmod \boldsymbol{p}^{m}$ satisfying $P f(x) \equiv \pi^{e} \bmod \boldsymbol{p}^{m}$ and such that $y$ in $x$ has $0, \cdots, 0, \pi^{j}$ as its coefficients. Since $P f(x) \equiv \pi^{e} \bmod p^{m}$ becomes $P f\left(x_{2 n-1}\right) \pi^{j} \equiv \pi^{e} \bmod p^{m}$ for such $x$, the number in question is equal to

$$
\left(q^{j}\right)^{(n-1)(2 n-3)} \boldsymbol{F}_{n-1}(m-j, e-j) \cdot\left(q^{m}\right)^{2 n-2} .
$$

If we put these together (and divide by $q^{m n(2 n-1)}$ ), we get

$$
f_{n}(m, e)=\left(1-q^{-(2 n-1)}\right) \sum_{j=0}^{e} q^{-(2 n-1) j} f_{n-1}(m-j, e-j) .
$$

This formula for $n \geqq 2$ and $f_{1}(m, e)=q^{-m}$ determine $f_{n}(m, e)$ uniquely. Therefore, we have only to show that the proposed expression for $f_{n}(m, e)$ satisfies the above recursion formula.

If we put $t=q^{-2}$, the identity to be verified becomes

$$
\left(1-t^{n-1}\right) \sum_{j=0}^{e} t^{(n-1) j} \prod_{i=1}^{n-2}\left(1-t^{i-j+e}\right)=\prod_{i=1}^{n-1}\left(1-t^{i+e}\right),
$$

in which $n \geqq 2$. Since this identity holds for $n=2$, we shall apply an induction on $n$ assuming that $n \geqq 3$. We observe that the left side is equal to

$$
\begin{aligned}
\sum_{j=0}^{e} t^{(n-1) j} & \prod_{i=1}^{n-2}\left(1-t^{i-j+e}\right) \\
& -\sum_{j=0}^{e} t^{(n-1)(j+1)} \prod_{i=1}^{n-2}\left(1-t^{i-j+e}\right) \\
= & \prod_{i=1}^{n-2}\left(1-t^{i+e}\right)+\sum_{j=1}^{e+1} t^{(n-1) j} \prod_{i=1}^{n-2}\left(1-t^{i-j+e}\right) \\
& -\sum_{j=1}^{e+1} t^{(n-1) j} \prod_{i=2}^{n-1}\left(1-t^{i-j+e}\right) \\
= & \prod_{i=1}^{n-2}\left(1-t^{i+e}\right)+\sum_{j=1}^{e+1} t^{(n-1) j} \prod_{i=2}^{n-2}\left(1-t^{i-j+e}\right) \text {-times } \\
= & \prod_{i=1}^{n-2}\left(1-t^{i+e}\right)-t^{n-1+e}-\operatorname{times} \\
& \left(1-t^{n-2}\right) \sum_{j=0}^{e} t^{(n-2) j} \prod_{i=1}^{n-3}\left(1-t^{i-j+e}\right)
\end{aligned}
$$

If we apply our induction assumption to the last expression, we see that the whole thing is equal to 


$$
\prod_{i=1}^{n-2}\left(1-t^{i+e}\right)-t^{n-1+e} \prod_{i=1}^{n-2}\left(1-t^{i+e}\right)=\prod_{i=1}^{n-1}\left(1-t^{i+e}\right) .
$$

This is the right side of the identity to be verified. q.e.d.

Lemma 5. If we put $g_{n}(m)=f_{n}(m, m)-f_{n}(m, m-1)$, we have

$$
g_{n}(m)=\sum_{j=1}^{n-1} f_{j}(3 m+2 j-2, m-1)
$$

with the understanding that the sum represents 0 for $n=1$.

Proof. First of all, by computing the number of cosets in $X^{0} / \boldsymbol{p}^{m} X^{0}$ in two different ways, we get

$$
\sum_{e=0}^{m-1} q^{m-e}\left(1-q^{-1}\right) F_{n}(m, e)+F_{n}(m, m)=q^{m n(2 n-1)} .
$$

We divide both sides by $q^{m n(2 n-1)}$; then, by putting $t=q^{-1}$, we get

$$
f_{n}(m, m)=1-(1-t) \sum_{e=0}^{m-1} t^{e-m} f_{n}(m, e) .
$$

If we substitute $f_{n}(m, e)$ by its expression given in Lemma 4 (after replacing $q^{-1}$ by $t$ ), we will get an expression for $f_{n}(m, m)$ in terms of $t$. In particular, we have $f_{1}(m, m)=t^{m}$, and hence $g_{1}(m)=0$; this coincides with the proposed expression for $g_{n}(m)$ in the case where $n=1$. Therefore, we shall assume that $n \geqq 2$, and put

$$
h_{n}(m)=g_{n}(m)-g_{n-1}(m) ;
$$

then we have

$$
\begin{aligned}
h_{n}(m)= & -(1-t) \sum_{e=0}^{m-1} t^{e-m}\left(f_{n}(m, e)-f_{n-1}(m, e)\right) \\
& -f_{n}(m, m-1)+f_{n-1}(m, m-1) .
\end{aligned}
$$

On the other hand, we have

$$
\begin{aligned}
& f_{n}(m, e)-f_{n-1}(m, e)=t^{m+2 n-2}\left(\prod_{i=1}^{n-2}\left(1-t^{2 i+1}\right)\left(1-t^{2 i}\right)^{-1}\left(1-t^{2(i+e)}\right)\right) \\
& \cdot\left(1-t^{2 n-2}\right)^{-1}\left(\left(1-t^{2 e}\right)-t\left(1-t^{2(n-1+e)}\right)\right) \\
&=t^{m+2 n-2} \prod_{i=1}^{n-2}\left(1-t^{2 i+1}\right) \cdot \prod_{i=1}^{n-1}\left(1-t^{2 i}\right)^{-1} \text {-times } \\
&\left(\prod_{i=1}^{n-1}\left(1-t^{2(i-1+e)}\right)-t \prod_{i=1}^{n-1}\left(1-t^{2(i+e)}\right)\right),
\end{aligned}
$$

and hence 


$$
\sum_{e=0}^{m-1} t^{e-m}\left(f_{n}(m, e)-f_{n-1}(m, e)\right)=-t^{2 n-2}\left(1-t^{2 n-1}\right)^{-1} f_{n}(m, m-1) .
$$

This implies that

$$
\begin{aligned}
h_{n}(m)= & t^{2 n-2}(1-t)\left(1-t^{2 n-1}\right)^{-1} f_{n}(m, m-1) \\
& -f_{n}(m, m-1)+f_{n-1}(m, m-1) \\
= & -\left(1-t^{2(m+n-2)}\right) f_{n-1}(m, m-1)+f_{n-1}(m, m-1) \\
= & t^{2(m+n-2)} f_{n-1}(m, m-1) .
\end{aligned}
$$

If we replace $n$ successively by $2, \cdots, n$, and take the corresponding sum, then we will get the formula stated in the lemma. q.e.d.

COROLlaRY. If we denote by $O\left(q^{-2}\right)$ a quantity such that $q^{2} \cdot O\left(q^{-2}\right)$ is bounded for all $m, q$, and $n$, then we have

$$
g_{n}(m)=q^{-3 m}\left(1+O\left(q^{-2}\right)\right) \quad(n \geqq 2) .
$$

In fact, we have

$$
\begin{aligned}
q^{2}\left(q^{3 m} g_{n}(m)-1\right) & =\sum_{j=2}^{n-1} q^{3 m+2} f_{j}(3 m+2 j-2, m-1) \\
& \leqq \sum_{j=0}^{\infty} 2^{-2 j} \cdot \prod_{i=1}^{\infty}\left(1-2^{-2 i}\right)^{-1}
\end{aligned}
$$

clearly, this infinite series and this infinite product are convergent.

4. Asymptotic estimates. In general, by a measure on a locally compact space $E$, we understand the Borel measure on $E$ determined by a positive linear functional on the vector space of $\mathbf{C}$-valued continuous functions on $E$ with compact supports. If $X$ is a locally compact commutative group and $X^{*}$ its dual, we shall use dual measures on $X, X^{*}$, i.e., Haar measures $d x, d x^{*}$ on $X, X^{*}$ such that, for every $\Phi$ in the Schwartz-Bruhat space $\mathscr{S}(X)$, the Fourier transformation $\Phi \rightarrow \Phi^{*}$ defined by

$$
\Phi^{*}\left(x^{*}\right)=\int_{X} \Phi(x)\left\langle x, x^{*}\right\rangle d x
$$

in which $\left\langle x, x^{*}\right\rangle=x^{*}(x)$, preserves the $L^{2}$-norms. In the special case where $X^{*}$ is bicontinuously isomorphic to $X$, we shall sometimes identify $X$ with $X^{*}$. In such case, we shall use the autodual measure on $X$, i.e., the unique Haar measure $d x$ on $X$ satisfying $d x=d x^{*}$. We recall that if $\Lambda(X)$ denotes the subspace of $L^{1}(X)$ consisting of all continuous $L^{1}$ - 
functions $\Phi$ with $\Phi^{*}$ in $L^{1}\left(X^{*}\right)$, the Fourier transformation gives a bijection of $\Lambda(X)$ to $\Lambda\left(X^{*}\right)$ satisfying $\left(\Phi^{*}\right)^{*}(x)=\Phi(-x)$ for every $x$ in $X$.

Suppose that $X, Y$ are locally compact commutative groups and $f: X \rightarrow Y$ a continuous mapping. We choose a Haar measure $d x$ on $X$ and dual measures $d y, d y^{*}$ on $Y, Y^{*}$. And, for every $\Phi$ in $L^{1}(X)$, we put

$$
F_{\Phi}^{*}\left(y^{*}\right)=\int_{X} \Phi(x)\left\langle f(x), y^{*}\right\rangle d x
$$

In this way, we get a bounded uniformly continuous function $F_{\Phi}^{*}$ on $Y^{*}$. We recall that the first sections of Weil [9] are devoted to proving certain general properties of this function. Among other things, he proved that if $f$ satisfies the "condition $(A)$ ", i.e., if $F_{\infty}^{*}$ is integrable on $Y^{*}$ uniformly in $\Phi$ restricted to a compact subset of $\mathscr{S}(X), F_{\phi}^{*}$ belong to $\Lambda\left(Y^{*}\right)$; moreover, there exists a unique family of measures $d \mu_{y}$ on $X$, each $d \mu_{y}$ being the image measure under $f^{-1}(y) \rightarrow X$ of a measure on $f^{-1}(y)$, such that the corresponding function in $\Lambda(Y)$ is given by

$$
F_{\Phi}(y)=\int_{X} \Phi(x) d \mu_{y}(x)
$$

We shall show that, in the local and the global cases, $f=P f$ satisfies the condition $(A)$. We shall use the following fact:

Let $X$ denote a locally compact commutative group and $\psi$ a nondegenerate second degree character of $X$, i.e., a continuous mapping of $X$ to $\mathbf{C}_{1}^{x}$, the multiplicative group of complex numbers of absolute value 1 , satisfying

$$
\psi(x+y)=\psi(x) \psi(y)\langle x, y h\rangle \quad(x, y \in X)
$$

for some bicontinuous isomorphism $h: X \rightarrow X^{*}$. Then the Fourier transform $\psi^{*}$ of $\psi$ is given by

$$
\psi^{*}\left(x^{*}\right)=\gamma(\psi)|h|^{-\frac{1}{2}} \psi\left(x^{*} h^{-1}\right)^{-1},
$$

in which $\gamma(\psi)$ is an element of $\mathbf{C}_{1}^{x}$ and $|h|$ the modulus of $h$. For the proof, we refer to Weil [8], p. 161.

Let $k_{v}$ denote a local field (of characteristic 0 ) and $\chi_{v}$ a nontrivial character of $k_{v}$; we identify $k_{v}$ with its dual by $\left(i, i^{*}\right) \rightarrow \chi_{v}\left(i i^{*}\right)$ for $i, i^{*}$ in $k_{v}$. Let $X$ denote the vector space of alternating matrices of degree $2 n$ and put $X_{v}=X_{k_{v}}$; we identify $X_{v}$ with its dual by $(x, y) \rightarrow \chi_{v}\left(\frac{1}{2} \operatorname{tr}\left(x^{t} y\right)\right)$ for $x, y$ in $X_{v}$. We shall denote the autodual measures on $k_{v}$ and $X_{v}$ by 
$|d i|_{v}$ and $|d x|_{v}$. In the case where $k_{v}$ is non-archimedian, we shall denote by $\boldsymbol{o}_{v}, \boldsymbol{p}_{v}$, and $q_{v}$ the maximal compact subring of $k_{v}$, the ideal of nonunits of $\boldsymbol{o}_{v}$, and the number of elements of $\boldsymbol{o}_{v} / \boldsymbol{p}_{v}$. We shall use Lemmas 4 and 5 by taking $\boldsymbol{o}_{v}, \boldsymbol{p}_{v}$ as $\boldsymbol{o}, \boldsymbol{p}$; we define $X_{v}^{0}$ as $X^{0}$. We shall denote by $d=d_{v}$ the largest integer such that $\boldsymbol{p}_{v}^{-d}$ is contained in the kernel of $\chi_{v}$ and by $m\left(X_{v}^{0}\right)$ the measure of $X_{v}^{0}$ with respect to $|d x|_{v}$.

LEMMA 6. If $i^{*}$ is an element of $k_{v}$ of order $-m$, we have

$$
m\left(X_{v}^{0}\right)^{-1} \int_{X_{v}^{0}} \chi_{v}\left(P f(x) i^{*}\right)|d x|_{v}=g_{n}(m-d) \quad \text { or } \quad 1
$$

according as $m>d$ or $m \leqq d$.

Proof. Since the case where $m \leqq d$ is trivial, we shall assume that $m>d$. For every $i$ in $o$, let $E_{i}$ denote the open subset of $X_{v}^{0}$ defined by $P f(x) \equiv i \bmod p^{m-d}$ and $m\left(E_{i}\right)$ the measure of $E_{i}$ with respect to $|d x|_{v}$; then we have $m\left(E_{i}\right)=f_{n}(m-d$, ord $(i)) m\left(X_{v}^{0}\right)$. Hence the left side is equal to

$$
\begin{aligned}
& m\left(X_{v}^{0}\right)^{-1} \sum_{i \bmod \boldsymbol{p}^{m-a}} \int_{E_{i}} \chi_{v}\left(P f(x) i^{*}\right)|d x|_{v} \\
& =\sum_{e=0}^{m-d} f_{n}(m-d, e)\left(\sum_{i \bmod \mathbf{p}^{m-d}, \text { ord }(i)=e} \chi_{v}\left(i i^{*}\right)\right) .
\end{aligned}
$$

By the orthogonality of characters, the sum of $\chi_{v}\left(i i^{*}\right)$ over the set of cosets $i+\boldsymbol{p}^{m-d}$ for $i$ in $\boldsymbol{p}^{m-d-1}$ is 0 . Therefore, the above sum is equal to $-f_{n}(m-d, m-d-1)+f_{n}(m-d, m-d)=g_{n}(m-d)$. q.e.d.

We take $\Phi$ from $\mathscr{S}\left(X_{v}\right)$ and consider the function $F_{\Phi}^{*}$ for $P f: X_{v} \rightarrow$ $k_{v}$, i.e., we put

$$
F_{\Phi}^{*}\left(i^{*}\right)=\int_{X_{v}} \Phi(x) \chi_{v}\left(\operatorname{Pf}(x) i^{*}\right)|d x|_{v}
$$

If $k_{v}$ is non-archimedian and if $\Phi$ is the characteristic function of $X_{v}^{0}$, Lemma 6 gives $F_{\Phi}^{*}$ explicitly. On the other hand, since $F_{\Phi}^{*}$ is just the Fourier transform of $\Phi$ for $n=1$, in the following we shall assume that $n \geqq 2$. We shall show that the case where $n=2$ is still exceptional.

In general, let $h$ denote a symmetric matrix of degree $m$ with coefficients in $k_{v}$ such that $\operatorname{det}(h) \neq 0$; put

$$
Q(z)=\frac{1}{2} z h^{t} z, \quad \psi(z)=\chi_{v}(Q(z))
$$


for every $z$ in $k_{v}^{m}=M_{1, m}\left(k_{v}\right)$. We identify $k_{v}^{m}$ with its dual by $\left(z, z^{\prime}\right) \rightarrow$ $\chi_{v}\left(z^{t} z^{\prime}\right)$; let $|d z|_{v}$ denote the autodual measure on $k_{v}^{m}$. Let $\left|i^{*}\right|_{v}$ for $i^{*}$ in $k_{v}^{\times}$denote the modulus of the automorphism of $k_{v}$ defined by $i \rightarrow i i^{*}$; put $|0|_{v}=0$. Then (1) implies that

$$
\left.\left.\left|\int_{k_{v}^{m}} \Phi(z) \psi(z)\right| d z\right|_{v}\left|\leqq\left\|\Phi^{*}\right\|_{1}\right| \operatorname{det}(h)\right|_{v} ^{-\frac{1}{2}} .
$$

Since the left side has $\|\Phi\|_{1}$ as its obvious upper bound, we get the following inequality:

$$
\left.\left|\int_{k_{v}^{m}} \Phi(z) \psi(z)\right| d z\right|_{v} \mid \leqq \max \left(\|\Phi\|_{1},\left\|\Phi^{*}\right\|_{1}\right) \min \left(1,|\operatorname{det}(h)|_{v}^{-\frac{1}{2}}\right) .
$$

If we identify $X_{v}$ for $n=2$ with $k_{v}^{6}$ and $P f(x)$ with $Q(z)$ so that we get $\operatorname{det}(h)=-1,\left(1^{\prime}\right)$ will give

$$
\left|F_{\Phi}^{*}\left(i^{*}\right)\right| \leqq \max \left(\|\Phi\|_{1},\left\|\Phi^{*}\right\|_{1}\right) \max \left(1,\left|i^{*}\right|_{v}\right)^{-3}
$$

for every $i^{*}$ in $k_{v}$.

We shall consider the general case where $n \geqq 2$. Let $y$ denote the submatrix of $x$ obtained from $x$ by crossing out its $(2 n-1)$-th and $2 n$-th rows and columns; let $Y_{v}$ denote the $y$-space. By Lemma 1 we have $P f(x)=\alpha \cdot P f(y)+Q_{y}(z)$, in which $Q_{y}(z)$ is a quadratic form on $Z_{v}=$ $k_{v}^{4 n-4}$ with $\operatorname{det}(y)^{2 n-4}$ as its discriminant. We identify $Y_{v}$ and $Z_{v}$ with their duals by $\left(y, y^{\prime}\right) \rightarrow \chi_{v}\left(\frac{1}{2} \operatorname{tr}\left(y^{t} y^{\prime}\right)\right)$ and $\left(z, z^{\prime}\right) \rightarrow \chi_{v}\left(z^{t} z^{\prime}\right)$; then $|d x|_{v}$ becomes the product of autodual measures $|d \alpha|_{v},|d y|_{v}$, and $|d z|_{v}$. We shall show that, for any compact subset $C$ of $\mathscr{S}\left(X_{v}\right)$, there exists an element $\Psi \geqq 0$ of $\mathscr{S}\left(Y_{v}\right)$ satisfying

$$
\left|F_{\Phi}^{*}\left(i^{*}\right)\right| \leqq \int_{Y_{v}} \Psi(y) \max \left(1,|P f(y)|_{v}^{n-2}\left|i^{*}\right|_{v}^{n-1}\right)^{-2}|d y|_{v}
$$

for every $\Phi$ in $C$. Let $\Phi_{1}, \Phi_{2}$ denote the partial Fourier transforms of $\Phi$ with respect to $|d \alpha|_{v},|d \alpha|_{v}|d z|_{v}$; then, by applying ( $\left.1^{\prime}\right)$ to

$$
F_{\Phi}^{*}\left(i^{*}\right)=\int_{Y_{v}}|d y|_{v}\left(\int_{z_{v}} \Phi_{1}\left(\operatorname{Pf}(y) i^{*}, y, z\right) \chi_{v}\left(Q_{y}(z) i^{*}\right)|d z|_{v}\right)
$$

we get

$$
\left|F_{\Phi}^{*}\left(i^{*}\right)\right| \leqq \int_{Y_{v}} \Theta(y) \max \left(1,|P f(y)|_{v}^{n-2}\left|i^{*}\right|_{v}^{n-1}\right)^{-2}|d y|_{v}
$$

in which 


$$
\Theta(y)=\int_{z_{v}} \max \left(\left|\Phi_{1}\right|,\left|\Phi_{2}\right|\right)\left(\operatorname{Pf}(y) i^{*}, y, z\right)|d z|_{v} .
$$

We observe that if $\Phi$ runs over $C, \Phi_{1}$ and $\Phi_{2}$ run over compact subsets of $\mathscr{S}\left(X_{v}\right)$. We shall separate two cases:

If $k_{v}$ is non-archimedian, there exists a positive integer $N$ such that the supports of $\Phi_{1}, \Phi_{2}$ are contained in $p^{-N} X_{v}^{0}$ for every $\Phi$ in $C$; also $\Phi_{1}$, $\Phi_{2}$ for $\Phi$ running over $C$ are uniformly bounded. Therefore, we can take as $\Psi$ a constant multiple of the characteristic function of $p^{-N} Y_{v}^{0}$. If $k_{v}$ is archimedian, the existence of $\Psi$ follows from the following lemma:

LEMma 7. Let $\mathbf{R}^{m}=\mathbf{R}^{m_{1}} \times \cdots \times \mathbf{R}^{m_{t}}$ denote a decomposition of $\mathbf{R}^{m}$ and $C$ a compact subset of $\mathscr{S}\left(\mathbf{R}^{m}\right)$. Then, there exists an element $\varphi \geqq 0$ of $\mathscr{S}(\mathbf{R})$ such that

$$
|\Phi(x)| \leqq \prod_{i=1}^{t} \varphi\left(r_{i}\left(x_{i}\right)\right)
$$

for every $\Phi$ in $C$ and $x=\left(x_{1}, \cdots, x_{t}\right)$ in $\mathbf{R}^{m}$, in which $r_{i}\left(x_{i}\right)$ is a distance function on $\mathbf{R}^{m_{i}}$ for $1 \leqq i \leqq t$.

This is a slight modification of Lemma 5 in [8], and it can be proved in the same way. We observe that we can take as $\Psi$ a function of the form $\varphi \circ r$, in which $\varphi \geqq 0$ is in $\mathscr{S}(\mathbf{R})$ and $r$ is a distance function on $Y_{v}$. We need the following lemma:

LEMMA 8. If $\sigma>1$, for every $t$ in $k_{v}^{\times}$, we have

$$
\int_{i_{v}} \max \left(|t|_{v},|i|_{v}\right)^{-\sigma}|d i|_{v}=\text { const. }|t|_{v}^{1-\sigma} .
$$

If we use the usual Haar measure on $k_{v}$ instead of $|d i|_{v}$, i.e., the Haar measure such that the unit disc is of measure 1,2 , or $2 \pi$ according as $k_{v}$ is non-archimedian, $k_{v}=\mathbf{R}$, or $k_{v}=\mathbf{C}$, the "const." becomes

$$
\left(1-q^{-\sigma}\right)\left(1-q^{1-\sigma}\right)^{-1}, 2 \sigma(\sigma-1)^{-1}, \quad \text { or } 2 \pi \sigma(\sigma-1)^{-1} \text {. }
$$

Since the proof is straightforward, we leave it as an exercise. The following is our principal lemma:

LEMMA 9. If $C$ is a compact subset of $\mathscr{S}\left(X_{v}\right)$, there exists a positive constant $c$ such that

$$
\left|F_{\Phi}^{*}\left(i^{*}\right)\right| \leqq c \cdot \max \left(1,\left|i^{*}\right|_{v}\right)^{-\sigma_{v}}
$$


for every $\Phi$ in $C$ and $i^{*}$ in $k_{v}$; we have $\sigma_{v}=3$ for $n=2$ and we can take $\sigma_{v}=(n-1)(n-2)^{-1}$ for $n \geqq 3$.

Proof. Since we have settled the case where $n=2$, we shall assume that $n \geqq 3$ and apply an induction on $n$. By Lemma 8 and by the induction assumption, $P f: Y_{v} \rightarrow k_{v}$ satisfies the condition $(A)$. Therefore, there exists a family of measures $d \nu_{i}$ on $Y_{v}$ such that

$$
F_{\Psi}^{*}\left(i^{*}\right)=\int_{Y_{v}} \Psi(y) \chi_{v}\left(\operatorname{Pf}(y) i^{*}\right)|d y|_{v}
$$

becomes the Fourier transform of

$$
F_{\Psi}(i)=\int_{Y_{v}} \Psi(y) d \nu_{i}(y)
$$

moreover, we have

$$
\left|F_{\Phi}^{*}\left(i^{*}\right)\right| \leqq \int_{k_{v}} F_{\psi}(i) \max \left(1,|i|_{v}^{n-2}\left|i^{*}\right|{ }_{v}^{n-1}\right)^{-2}|d i|_{v} .
$$

The right side has $\left\|F_{\Psi}\right\|_{1}$ as its obvious upper bound. On the other hand, it is at most equal to

$$
\left\|F_{\Psi}\right\|_{\infty} \cdot \int_{k_{v}} \max \left(1,|i|_{v}^{n-2}\left|i^{*}\right|_{v}^{n-2}\right)^{-2}|d i|_{v} .
$$

The above elementary integral can be calculated by Lemma 8 , and we get const. $\left|i^{*}\right|_{v}^{-\sigma}$ for $\sigma=(n-1)(n-2)^{-1}$. By putting the two estimates together, we get

$$
\left|F_{\Phi}^{*}\left(i^{*}\right)\right| \leqq \text { const. } \max \left(\left\|F_{\Psi}\right\|_{1},\left\|F_{\Psi}\right\|_{\infty}\right) \max \left(1,\left|i^{*}\right|_{v}\right)^{-\sigma} .
$$

q.e.d.

Remark. Lemmas 8 and 9 show that $P f: X_{v} \rightarrow k_{v}$ satisfies the condition $(A)$. As we shall see, Lemmas 6 and 9 are sufficient to prove that $P f$ satisfies the condition $(A)$ also in the global case. Nevertheless, it is an interesting problem to examine whether we have $\sigma_{v}=3$ for every $n \geqq 2$. If $k_{v}$ is non-archimedian, it is easy to show that the answer is affirmative. Therefore, the problem exists only in the archimedian case.

Since $P_{f}: X_{v} \rightarrow k_{v}$ satisfies the condition $(A)$, there exists a unique family of measures $d \mu_{i}$ on $X_{v}$, each $d \mu_{i}$ having its support in $(P f)^{-1}(i)$, such that 


$$
\int_{X_{v}} \Phi(x)|d x|_{v}=\int_{k_{v}}\left(\int_{X_{v}} \Phi(x) d \mu_{i}(x)\right)|d i|_{v}
$$

for every $\Phi$ in $\mathscr{S}\left(X_{v}\right)$. On the other hand, for every $i$ in $k_{v}$, the gauge form $\theta_{i}$ on $U(i)$ determines a measure $\left|\theta_{i}\right|_{v}$ on $U(i)_{v}=U(i)_{k_{v}}$.

LEMMA 10. The measure $d \mu_{i}$ is the image measure of $\left|\theta_{i}\right|_{v}$ under $U(i)_{v} \rightarrow X_{v}$ for every $i$ in $k_{v}$.

Proof. The fact that $d \mu_{i}$ is the image measure of $\left|\theta_{i}\right|_{v}$ for $i \neq 0$ follows from the remark on pp. 13-14 in Weil [9]. The point is that $d \mu_{0}$ is also the image measure of $\left|\theta_{0}\right|_{v}$. For the same reason as above, the restriction of $d \mu_{0}$ to $\left(U_{n-1}\right)_{v}$ coincides with $\left|\theta_{0}\right|_{v}$; according to Lemma 16 in Weil, op. cit., $d \mu_{0}$ can be expressed uniquely as the sum of the image measures of measures on $\left(U_{0}\right)_{v}, \cdots,\left(U_{n-1}\right)_{v}$. Let $d \mu$ denote the image measure of the measure on $\left(U_{r}\right)_{v}$; we have only to show that $d \mu=0$ for $r \neq n-1$. We use the action of $G^{*}=G L_{2 n}\left(k_{v}\right)$ on $X_{v}$ and manipulate (2) as in Weil, loc. cit.; then we get

$$
d \mu_{t i}\left(g x^{t} g\right)=|t|_{v}^{2(n-1)} d \mu_{i}(x),
$$

in which $t=\operatorname{det}(g)$. Since $\left(U_{0}\right)_{v}, \cdots,\left(U_{n-1}\right)_{v}$ are invariant under the action of $G^{*}$, by taking 0 as $i$, we get

$$
d \mu\left(g x^{t} g\right)=|t|_{v}^{2(n-1)} d \mu(x)
$$

for every $g$ in $G^{*}$. On the other hand, let $H^{*}$ denote the stabilizer of $G^{*}$ at the representative of $\left(U_{r}\right)_{v}$ composed of $e_{r}, 0,0,0$; then, as we have seen in the proof of Lemma $3, H^{*}$ consists of

$$
g=\left[\begin{array}{ll}
u_{1} & u_{12} \\
0 & u_{2}
\end{array}\right]
$$

in which $u_{1}$ is in $S p_{2 r}\left(k_{v}\right), u_{2}$ in $G L_{2(n-r)}\left(k_{v}\right)$, and $u_{12}$ in $M_{2 r, 2(n-r)}\left(k_{v}\right)$. Therefore, if we put $\omega(g)=d u_{1} \wedge d u_{2} \wedge d u_{12}$, for every $a$ in $H^{*}$ we have $\omega\left(a^{-1} g a\right)=\operatorname{det}(a)^{2 r} \omega(g)$. Hence, if $d \mu \neq 0$, by the theory of relatively invariant measures, we get $|\operatorname{det}(a)|_{v}^{2(n-1)}=|\operatorname{det}(a)|_{v}^{2 r}$ for every $a$ in $H^{*}$. This implies that $r=n-1$. q.e.d.

5. A dominant series. We shall prove the convergence of a certain series which will turn out to be a dominant series for the EisensteinSiegel series. We shall start by proving the following elementary lemma: 
LEMMA 11. Let $k_{v}$ denote an archimedian local field, dt the usual Haar measure on $k_{v}$ normalized by the condition that the unit dise in $k_{v}$, defined by $|t|_{v} \leqq 1$, is of measure 2 or $2 \pi$ according as $k_{v}=\mathbf{R}$ or $k_{v}=\dot{\mathbf{C}}$; suppose that $\alpha, \delta>0$ and $t_{0}, t_{1}$ elements of $k_{v}$, in which $\left|t_{0}\right|_{v} \geqq \delta$. Then there exists a positive constant $c_{0}$, for which we can take $\min (1,2 \delta)$ or $(\pi / 2) \min (1,4 \delta)$ according as $k_{v}=\mathbf{R}$ or $k_{v}=\mathbf{C}$, such that

$$
\int_{\left|t-t_{1}\right|_{v} \leqq 1} \max \left(\left|t_{0}\right|_{v},|t|_{v}\right)^{-\alpha} d t \geqq c_{0} \cdot \max \left(\left|t_{0}\right|_{v},\left|t_{1}\right|_{v}\right)^{-\alpha} .
$$

Proof. For the sake of simplicity, we put $t_{0}=a, t_{1}=b$; also, we shall denote by $C$ and $C^{\prime}$ the subsets of $k_{v}$ defined respectively by $|t-b|$ $\leqq 1$ and $|t| \leqq|a|$. We are supposed to show that

$$
I=\int_{C} \max \left(|a|_{v},|t|_{v}\right)^{-\alpha} d t
$$

is at least equal to $c_{0} \cdot \max \left(|a|_{v},|b|_{v}\right)^{-\alpha}$ provided that $|a|_{v} \geqq \delta$. Suppose first that $|b| \geqq \frac{1}{2}$; then $r=1-(2|b|)^{-1} \geqq 0$. Let $C^{\prime \prime}$ denote the subset of $k_{v}$ defined by $|t-r b| \leqq \frac{1}{2}$; then $C^{\prime \prime}$ is contained in the subset of $C$ defined by $|t-b| \leqq 1,|t| \leqq|b|$. Therefore, on $C^{\prime \prime}$ we have $\max \left(|a|_{v},|t|_{v}\right)^{-\alpha}$ $\geqq \max \left(|a|_{v},|b|_{v}\right)^{-\alpha}$; hence

$$
I \geqq \max \left(|a|_{v},|b|_{v}\right)^{-\alpha} \cdot m\left(C^{\prime \prime}\right),
$$

in which $m\left(C^{\prime \prime}\right)$ is the measure of $C^{\prime \prime}$ with respect to $d t$. We have $m\left(C^{\prime \prime}\right)=1$ for $k_{v}=\mathbf{R}$ and $m\left(C^{\prime \prime}\right)=\pi / 2$ for $k_{v}=\mathbf{C}$. Suppose next that $|b| \leqq \frac{1}{2}$; let $C^{\prime \prime}$ denote the intersection of $C$ and $C^{\prime}$. Then, on $C^{\prime \prime}$ we have $\max \left(|a|_{v},|t|_{v}\right)^{-\alpha}=|a|_{v}^{-\alpha} \geqq \max \left(|a|_{v},|b|_{v}\right)^{-\alpha}$; hence

$$
I \geqq \max \left(|a|_{v},|b|_{v}\right)^{-\alpha} \cdot m\left(C^{\prime \prime}\right) \text {. }
$$

If $C^{\prime}$ is contained in $C$, we have $m\left(C^{\prime \prime}\right)=m\left(C^{\prime}\right)=2|a|_{v} \geqq 2 \delta$ for $k_{v}=\mathbf{R}$ and $m\left(C^{\prime \prime}\right)=2 \pi|a|_{v} \geqq 2 \pi \delta$ for $k_{v}=\mathbf{C}$. If $C^{\prime}$ is not contained in $C$, we have $|a|>1-|b| \geqq \frac{1}{2}$. Therefore $C^{\prime \prime}$ contains the subset of $k_{v}$ defined by $|t| \leqq \frac{1}{2}$; hence $m\left(C^{\prime \prime}\right) \geqq 1$ for $k_{v}=\mathbf{R}$ and $m\left(C^{\prime \prime}\right) \geqq \pi / 2$ for $k_{v}=\mathbf{C}$. Therefore, we can take $\min (1,2 \delta)$ as $c_{0}$ for $k_{v}=\mathbf{R}$ and $\min (\pi / 2,2 \pi \delta)=$ $(\pi / 2) \min (1,4 \delta)$ as $c_{0}$ for $k_{v}=\mathbf{C}$. q.e.d.

Lemma 11 will be used in the proof of the following equally elementary lemma:

LEMMA 12. Let $L_{i}(x)=a_{i 1} x_{1}+\cdots+a_{i d} x_{d}$ for $1 \leqq i \leqq d$ denote 
linear forms on $\mathbf{R}_{d}$, which are linearly independent over $\mathbf{C}$, such that $L_{i}(x)$ for $1 \leqq i \leqq r_{1}$ have real coefficients and $L_{r_{1}+j}(x), L_{r_{1}+r_{2}+j}(x)$ for $1 \leqq$ $j \leqq r_{2}$ have conjugate complex coefficients; let $\left(\lambda_{1} \cdots \lambda_{d}\right)$ denote a variable point of $\left(\mathbf{R}_{+}^{\times}\right)^{d}$ satisfying $\lambda_{r_{1}+j}=\lambda_{r_{1}+r_{2}+j}$ for $1 \leqq j \leqq r_{2}$; for any $\alpha>1$, consider the function $\varphi \geqq 0$ on $\mathbf{R}^{d}$ defined by

$$
\varphi(x)=\prod_{i=1}^{d} \max \left(\lambda_{i},\left|L_{i}(x)\right|\right)^{-\alpha} .
$$

Then, if we restrict $\lambda_{1}, \cdots, \lambda_{d}$ by $\lambda_{i} \geqq$ const. $>0$, there exists a positive constant $c$, which is independent of $\lambda_{1}, \cdots, \lambda_{d}$, such that

$$
\sum_{n \in \boldsymbol{Z}^{d}} \varphi(n) \leqq c \cdot\left(\prod_{i=1}^{d} \lambda_{i}\right)^{1-\alpha} .
$$

Proof. We shall first calculate the integral of $\varphi$ over $\mathbf{R}^{d}$ with respect to the usual Haar measure $d x$. We put $y_{i}=L_{i}(x)$ for $1 \leqq i \leqq d$ and regard $y_{r_{1+j}}, y_{r_{1}+r_{2}+j}$ as (conjugate complex) points of $\mathbf{C}$. In this way, we get an $\mathbf{R}$-linear bijection of $\mathbf{R}^{d}$ to $\mathbf{R}^{r_{1}} \times \mathbf{C}^{r_{2}}$; we call them the $x$-space and the $y$-space. Let $a$ denote the coefficient-matrix of the linear transformation; then we have.

$$
\int_{\boldsymbol{R}^{d}} \varphi(x) d x=|\operatorname{det}(a)|^{-1}\left(2 \alpha(\alpha-1)^{-1}\right)^{r_{1}+r_{2}} \pi^{r_{2}} \cdot\left(\prod_{i=1}^{d} \lambda_{i}\right)^{1-\alpha} .
$$

Consider the image, say $V$, under $y=a x$ of the subset of the $x$-space defined by $|x|_{\infty}=\max \left(\left|x_{1}\right|, \cdots,\left|x_{d}\right|\right) \leqq 1$; then $V$ is a neighborhood of 0 in the $y$-space. Therefore, if we take $N$ sufficiently large, the image of the set defined by $|x|_{\infty} \leqq N$ contains the set defined by $|y|_{\infty} \leqq 1$. We take as $N$ the smallest positive integer satisfying this condition; $N$ depends only on $a$. Let $n$ denote an arbitrary element of $\mathbf{Z}^{d}$; then, by a simple calculation depending on Lemma 11, we get

$$
\int_{|x-n|_{\infty} \leqq N} \varphi(x) d x \geqq c_{1} \cdot \varphi(n) .
$$

In fact, if the condition on $\lambda_{1}, \cdots, \lambda_{d}$ is of the form $\lambda_{i} \geqq \delta>0$ for $1 \leqq$ $i \leqq r_{1}$ and $\lambda_{r_{1+j}} \geqq \delta^{\frac{1}{2}}$ for $1 \leqq j \leqq r_{2}$, the calculation shows that we can take

$$
c_{1}=|\operatorname{det}(\alpha)|^{-1} \min (1,2 \delta)^{r_{1}}((\pi / 2) \min (1,4 \delta))^{r_{2}} .
$$

Therefore, by taking the summation over $\mathbf{Z}^{d}$, we get 


$$
(2 N)^{d} \int_{\boldsymbol{R}^{d}} \varphi(x) d x \geqq c_{1} \cdot \sum_{n \in \mathbf{Z}^{d}} \varphi(n) ;
$$

hence the lemma holds with

$$
c=(4 N)^{d}\left(\alpha(\alpha-1)^{-1}\right)^{r_{1}+r_{2}} \min (1,2 \delta)^{-r_{1}} \min (1,4 \delta)^{-r_{2}} .
$$

q.e.d.

Let $k$ denote an algebraic number field and $o$ the ring of integers of $k$; choose a Z-base $a_{1}, \cdots, a_{d}$ of $\boldsymbol{o}$. We know that there are $d=$ [k:Q] distinct homomorphisms of $k$ to $\mathbf{C}$; the corresponding images $\left(a_{i 1} \cdots a_{i d}\right)$ of $\left(a_{1} \cdots a_{d}\right)$ for $1 \leqq i \leqq d$ can be so arranged that the matrix $a$ satisfies the condition in Lemma 12. We need two basic theorems in number theory: one states that the ideal class group of $k$ is finite. Let $S_{\infty}$ denote the set of archimedian valuations on $k$. Then, another states that if we map the unit group of $\boldsymbol{o}$ to $\left(\mathbf{R}_{+}^{\times}\right)^{r_{1}+r_{2}}$ as $u \rightarrow\left(|u|_{v}\right)_{v \in S_{\infty}}$, the image group forms a lattice in the subgroup defined by the condition that the product of all coordinates is 1 . Consequently, for any $b$ in $k^{\times}$ such that the product of all coordinates in $\left(\mathbf{R}_{+}^{\times}\right)^{r_{1}+r_{2}}$ is at least equal to 1 , there exists a unit $u$ of $o$ satisfying $|u b|_{v} \geqq \delta>0$ for every $v$ in $S_{\infty}$, in which $\delta$ depends only on $k$.

Proposition 1. Let $v$ denote a valuation on $k, n$ a non-negative integer, and $\varepsilon$ a positive real number; suppose that $\sigma_{v}>n$ for all $v$ and $\sigma_{v} \geqq n+1+\varepsilon$ for almost all $v$. Then the series

$$
\sum_{i} \prod_{v} \max \left(1,\left|i_{1}\right|_{v}, \cdots,\left|i_{n}\right|_{v}\right)^{-\sigma_{v}},
$$

in which $i=\left(i_{1} \cdots i_{n}\right)$ runs over $k^{n}$, is convergent.

Proof. Since the convergence is clear for $n=0$, we shall assume that $n \geqq 1$ and apply an induction on $n$. Let $E$ denote a subset of $\{1, \cdots, n\}$ and $k_{E}$ the subset of $k^{n}$ consisting of those $i$ in which $i_{p} \neq 0$ for every $p$ in $E$ and $i_{p}=0$ for every other $p$; then $k^{n}$ becomes the disjoint union of $k_{E}$ for all $E$. By the induction assumption, the partial sum over $k_{E}$ is convergent for every $E \neq\{1, \cdots, n\}$. Therefore we have only to prove the convergence of the partial sum over $\left(k^{\times}\right)^{n}$. By assumption, there exist real numbers $\alpha, \beta$ and a finite set $S$ of valuations on $k$ satisfying $\beta>n+1>\alpha>n$ such that $\sigma_{v} \geqq \alpha$ for all $v$ and $\sigma_{v} \geqq \beta$ for all $v$ not in $S$. We can replace $S$ by any larger set without changing 
$\alpha, \beta$. Suppose that $\boldsymbol{r}_{1}, \cdots, \boldsymbol{r}_{h}$ form a complete set of representatives of the ideal class group of $k$; we may assume that they are integral ideals. We shall assume that $S$ contains all prime factors of $\boldsymbol{r}_{1}, \cdots, \boldsymbol{r}_{h}$ as well as the set $S_{\infty}$. We observe that, as a function of $\sigma, \max \left(1,\left|i_{1}\right|_{v}, \cdots,\left|i_{n}\right|_{v}\right)^{-\sigma}$ is monotone decreasing. Therefore, we have only to prove the convergence in the case where $\sigma_{v}=\alpha$ for all $v$ in $S$ and $\sigma_{v}=\beta$ for all $v$ not in $S$. Let $i=\left(i_{1} \cdots i_{n}\right)$ denote an arbitrary element of $\left(k^{\times}\right)^{n}$; then there exists a set of integral ideals $\boldsymbol{a}_{1}, \cdots, \boldsymbol{a}_{n}, \boldsymbol{b}$ satisfying $\boldsymbol{b} i_{p}=\boldsymbol{a}_{p}$ for $1 \leqq p \leqq n$. We can assume that $\boldsymbol{a}_{1}, \cdots, \boldsymbol{a}_{n}, \boldsymbol{b}$ are relatively prime; then the set is unique. Moreover, there exists a unique index $j$ such that $\boldsymbol{b r}_{j}=\boldsymbol{o} b$ for some $b \neq 0$ in $\boldsymbol{o}$; put $b i_{p}=a_{p}$; then we have $\boldsymbol{a}_{p} \boldsymbol{r}_{j}=\boldsymbol{o} a_{p}$, hence $a_{p} \neq 0$ is in $o$, for every $p$. Since we can multiply any unit of $o$ to $b$, we may assume that $|b|_{v} \geqq \delta>0$ for every $v$ in $S_{\infty}$, in which $\delta$ depends only on $k$.

We observe that if a power $\boldsymbol{p}^{e}$ of a prime ideal $\boldsymbol{p}$ of $\boldsymbol{o}$ divides $a_{1}, \cdots, a_{n}, b$, it divides $\boldsymbol{r}_{j}$; hence $\boldsymbol{p}$ is in $S$. Let $\boldsymbol{p}^{e}$ denote the highest power of $\boldsymbol{p}$ which divides at least one of the $\boldsymbol{r}_{1}, \cdots, \boldsymbol{r}_{h}$ and put

$$
c^{\prime}=\prod_{\boldsymbol{p} \in S}\left(N_{\boldsymbol{p}}\right)^{\alpha e},
$$

in which $N$ denotes the norm. Then, by using the product-formula, we get

$$
\begin{aligned}
& \prod_{v} \max \left(1,\left|i_{1}\right|_{v}, \cdots,\left|i_{n}\right|_{v}\right)^{-\sigma_{v}} \\
& \quad=\prod_{v}|b|_{v}^{\sigma_{v}} \max \left(|b|_{v},\left|a_{1}\right|_{v}, \cdots,\left|a_{n}\right|_{v}\right)^{-\sigma_{v}} \\
& \quad \leqq c^{\prime} \cdot\left(\prod_{v \in S}|b|_{v}\right)^{\alpha-\beta}\left(\prod_{v \in S_{\infty}} \max \left(|b|_{v},\left|a_{1}\right|_{v}, \cdots,\left|a_{n}\right|_{v}\right)^{-\alpha} .\right.
\end{aligned}
$$

Therefore it is enough to show that the sum of the right side for $\left(a_{1} \cdots a_{n}\right)$ running over $\boldsymbol{o}^{n}$ and $\boldsymbol{o} b$ over the set of non-zero principal ideals of $\boldsymbol{o}$ is convergent. We recall that we have selected a unique generator $b$ of $o b$ satisfying $|b|_{v} \geqq \delta$ for every $v$ in $S_{\infty}$. Since we have $a>n$, we can apply Lemma 12 repeatedly, and we get

$$
\begin{aligned}
& \sum_{\left(a_{1} \cdots a_{n}\right) \in \boldsymbol{o}^{n}} \prod_{v \in S_{\infty}} \max \left(|b|_{v},\left|a_{1}\right|_{v}, \cdots,\left|a_{n}\right|_{v}\right)^{-\alpha} \\
& \leqq \text { const } \cdot\left(\prod_{v \in S_{\infty}}|b|_{v}\right)^{n-\alpha} .
\end{aligned}
$$

Therefore we have only to show that the series

$$
\sum_{o b}\left(\prod_{v \in S}|b|_{v}\right)^{\alpha-\beta}\left(\prod_{v \in S_{\infty}}|b|_{v}\right)^{n-\alpha}
$$


is convergent. Again by the product-formula, this can be written as

$$
\sum_{\boldsymbol{o} b}\left(\prod_{v \in S-S_{\infty}}|b|_{v}\right)^{\alpha-n}\left(\prod_{v \in S}|b|_{v}\right)^{\beta-n} .
$$

We observe that this series is at most equal to the product of $\left(1-\left(N_{p}\right)^{-(\alpha-n)}\right)^{-1}$ for all $\boldsymbol{p}$ in $S$ multiplied by the product of $\left(1-\left(N_{p}\right)^{-(\beta-n)}\right)^{-1}$ for all $p$ not in $S$. We recall finally that the sum of $(\mathrm{Na})^{-\sigma}$ over the set of all integral ideals $a$ of $k$ is convergent for $\sigma>1$. q.e.d.

Remark. If $i$ is a point of the projective space $P_{n}(k)$ and $\left(i_{0}, i_{1}, \cdots, i_{n}\right)$ a representative of $i$,

$$
H(i)=\prod_{v} \max \left(\left|i_{0}\right|_{v},\left|i_{1}\right|_{v}, \cdots,\left|i_{n}\right|_{v}\right)
$$

does not depend on the choice of the representative; it is called the height of $i$ [cf. 4]. As a corollary of Proposition 1, we get the wellknown fact stating that the series

$$
\sum_{i \in P_{n}(k)} H(i)^{-\sigma}
$$

is convergent for $\sigma>n+1$; see also [5], pp. 133-134.

6. The Siegel formula. We go back to the representation $\rho$ of $G=$ $S L_{m}$ in the vector space $X$ of alternating matices of degree $m \geqq 2$ defined over $k$ by $\rho(g) \cdot x=g x^{t} g$; we take as $k$ an algebraic number field. Let $d g$ denote a gauge form on $G$ defined over $k$ and $|d g|_{A}$ the corresponding "Tamagawa measure" on the adelization $G_{A}$ of $G$; this is an intrinsically defined (bi-invariant) Haar measure on $G_{A}$. We know that, for every $\Phi$ in the Schwartz-Bruhat space $\mathscr{S}\left(X_{A}\right)$, the integral

$$
I(\Phi)=\int_{G_{A} / G_{k}}\left(\sum_{\xi \in X_{k}} \Phi(\rho(g) \cdot \xi)\right)|d g|_{A}
$$

is absolutely convergent [cf. 3]. We have seen that every $G$-orbit in $X$ defined over $k$ contains a $k$-rational point; hence it is of the form $U=$ $\rho(G) \cdot \xi$ for some $\xi$ in $X_{k}$. We also recall that $U$ satisfies the Witt condition relative to any field; that the stabilizer of $G$ at $\xi$ is unimodular and has 1 as its "Tamagawa number". Let $\theta$ denote a $G$-invariant gauge form on $U$ defined over $k$ and $|\theta|_{A}$ the corresponding Tamagawa measure on $U_{A}$; then, by a standard argument [cf. 7, pp. 24-31; 9, pp. 14-16], we get 


$$
I(\Phi)=\sum_{U} \int_{U_{\boldsymbol{A}}} \Phi \cdot|\theta|_{A}
$$

In the case where $m$ is even, the set of $U$ 's contains $U(i)$ for all $i$ in $k$ as its members; the remaining part consists of $U_{r}$ for $0 \leqq r<\frac{1}{2} m-1$. In the case where $m$ is odd, the set of $U$ 's consists of $U(0)$ and $U_{r}$ for the same values of $r$. We observe that, for $U=U(i)$, we can take the residue $\theta_{i}$ of $(P f(x)-i)^{-1} d x$ on $U(i)$ as $\theta$; we shall denote the $\theta$ for $U=U_{r}$ by $\omega_{r}$. In the case where $m$ is odd, the above identity is the Siegel formula with the integral of $\Phi$ over $U(0)$ as the (incomplete) Eisenstein-Siegel series. In the case where $m=2 n$, because of the existence of an invariant, we will get a Siegel formula with a non-trivial Eisenstein-Siegel series.

Let $\chi$ denote a non-trivial character of $k_{A} / k$; we identify $k_{A}$ with its dual by $\left(i, i^{*}\right) \rightarrow \chi\left(i i^{*}\right)$ for $i, i^{*}$ in $k_{A}$; we put $[x, y]=\frac{1}{2} \operatorname{tr}\left(x^{t} y\right)$ and identify $X_{A}$ with its dual by $(x, y) \rightarrow \chi([x, y])$ for $x, y$ in $X_{A}$. Then the autodual measure $|d x|_{A}$ on $X_{A}$ is the Haar measure with respect to which $X_{A} / X_{k}$ is of measure 1. For every $\Phi$ in $\mathscr{S}\left(X_{A}\right)$, we define a function $F_{\Phi}^{*}$ on $k_{A}$ as

$$
F_{\Phi}^{*}\left(i^{*}\right)=\int_{X_{A}} \Phi(x) \chi\left(P f(x) i^{*}\right)|d x|_{A}
$$

Let $\chi_{v}$ denote the $v$-component of $\chi$, i.e., the character of the $v$-completion $k_{v}$ of $k$ defined as the product of the canonical injection $k_{v} \rightarrow k_{A}$ and $\chi$; then, for almost all $v$, the usual Haar measure on $k_{v}$ is autodual. Hence, for almost all non-archimedian $v, o_{v}$ is the kernel of $\chi_{v}$ and $m\left(X_{v}^{0}\right)=1$. We recall that $X_{A}$ is the inductive limit of $X_{S}=X_{0}^{0} \times X_{1}$, in which

$$
X_{0}^{0}=\prod_{v \notin S} X_{v}^{0}, \quad X_{1}=\prod_{v \in S} X_{v},
$$

for $S$ running over the family of finite sets of valuations on $k$ containing $S_{\infty}$. Therefore, for every compact subset $C$ of $\mathscr{S}\left(X_{A}\right)$, there exist an $S$ and a compact subset $C_{1}$ of $\mathscr{S}\left(X_{1}\right)$ such that every $\Phi$ in $C$ is of the form $\Phi_{0} \otimes \Phi_{1}$, in which $\Phi_{0}$ is the characteristic function of $X_{0}^{0}$ and $\Phi_{1}$ is in $C_{1}$. Put $\sigma_{v}=3$ if $n=2$ or $v \notin S$ and $\sigma_{v}=(n-1)(n-2)^{-1}$ if $n \geqq 3$ and $v \in S$; then, there exists a positive constant $c$ such that

$$
\sum_{i^{*} \in k}\left|F_{\Phi}^{*}\left(i^{*}\right)\right| \leqq c \cdot \sum_{i^{*} \in k} \prod_{v} \max \left(1,\left|i^{*}\right|_{v}\right)^{-\sigma_{v}}
$$


for every $\Phi$ in $C$. This can be proved easily by using Lemmas 6, 9 and the Fubini theorem. By Proposition 1, the right side is convergent. On the other hand, the mapping $\mathscr{S}\left(X_{A}\right) \times k_{A} \rightarrow \mathscr{S}\left(X_{A}\right)$ defined by $\left(\Phi, i^{*}\right)$ $\rightarrow \Phi_{i^{*}}$, in which $\Phi_{i^{*}}(x)=\Phi(x) \chi\left(P f(x) i^{*}\right)$, is continuous. The proof is straightforward. Therefore, by [9], pp. 7-8, the continuous mapping Pf: $X_{A} \rightarrow k_{A}$ satisfies the condition $(A)$, and we have the Poisson formula:

$$
\sum_{i * k} F_{\Phi}^{*}\left(i^{*}\right)=\sum_{i \in k}\left(F_{\Phi}^{*}\right) *(i)
$$

Put $\left(F_{\Phi}^{*}\right)^{*}(-i)=F_{\Phi}(i)$ for every $i$ in $k_{A}$; then we have

$$
F_{\Phi}(i)=\int_{U(i) \boldsymbol{A}} \Phi \cdot\left|\theta_{i}\right|_{A}
$$

for every $i$ in $k$. In order to prove this, we may restrict $\Phi$ to any subset of $\mathscr{S}\left(X_{A}\right)$ which spans a dense subspace of $\mathscr{S}\left(X_{A}\right)$. We take as $\Phi$ the product of $\Phi_{v}$ in which $\Phi_{v}$ is in $\mathscr{S}\left(X_{v}\right)$ for every $v$ and is the characteristic function of $X_{v}^{0}$ for every non-archimedian $v$ except for a finite number of $v$ 's. Then $F_{\Phi}$ decomposes into the product of $F_{\Phi_{v}}$ defined by $F_{\Phi_{v}}\left(i_{v}\right)=\left(F_{\Phi_{v}}^{*}\right) *\left(-i_{v}\right)$, and by Lemma 10 we have

$$
F_{\Phi_{v}}\left(i_{v}\right)=\int_{U\left(i_{v}\right)_{v}} \Phi_{v} \cdot\left|\theta_{i_{v}}\right|_{v}
$$

for every $i_{v}$ in $k_{v}$. This implies the assertion. We have thus obtained the following theorem:

THEOREM 1. If $m=2 n$, i.e., if $G=S L_{2 n}$, for every $\Phi$ in $\mathscr{S}\left(X_{A}\right)$, we have $I(\Phi)=E(\Phi)$, in which

$$
E(\Phi)=\sum_{i^{*} \in k} \int_{X_{A}} \Phi(x) \chi\left(P f(x) i^{*}\right)|d x|_{A}+\sum_{r=0}^{n-2} \int_{\left(U_{r}\right)_{A}} \Phi \cdot\left|\omega_{r}\right|_{A} \cdot
$$

In the case where $n=1$, if we identify $x$ with $\operatorname{Pf}(x)=x_{12}, I(\Phi)=$ $E(\Phi)$ reduces to the usual Poisson formula:

$$
\sum_{i \in k} \Phi(i)=\sum_{i^{*} \in k} \Phi^{*}\left(i^{*}\right) ;
$$

in the case where $n=2, I(\Phi)=E(\Phi)$ reduces to the Siegel formula for the quadratic form $P f(x)=x_{12} x_{34}-x_{13} x_{24}+x_{14} x_{23}$; the case where $n \geqq 3$ seems to be new.

7. The zeta function. We shall discuss the zeta function of $P f$ : we shall see that the functional equation of the zeta function is not as 
"deep" as the Siegel formula-it does not depend on the Poisson formula (4) involving elements of $\Lambda\left(k_{A}\right)$; it depends only on (3) and the usual Poisson formula involving elements of $\mathscr{S}\left(X_{A}\right)$.

Let $Y$ denote the Zariski open subset of $X$ defined over $k$ by $\operatorname{Pf}(x)$ $\neq 0$; then $G^{*}=G L_{2 n}$ acts transitively on $Y$ as $\rho\left(g^{*}\right) \cdot x=g^{*} x^{t} g^{*}$ with $S p_{2 n}$ as the stabilizer at $e_{n} ; \omega(x)=P f(x)^{-(2 n-1)} d x$ is a $G^{*}$-invariant gauge form on $Y$ defined over $k$. Let $v$ denote a non-archimedian valuation on $k$ and $Y_{v}^{0}$ the subset of $X_{v}^{0}$ defined by $|P f(x)|_{v}=1$; then, e.g., by Lemma 4 we have

$$
\int_{Y_{v}^{0}}|P f(x)|_{v}^{-(2 n-1)}|d x|_{v}=m\left(Y_{v}^{0}\right)=\prod_{i=1}^{n}\left(1-q_{v}^{-(2 i-1)}\right)
$$

for almost all $v$; hence $\lambda=\left(\left(1-q_{v}^{-1}\right)^{-1}\right)$ is a convergence factor for $Y$, and the Tamagawa measure $|\lambda \omega(x)|_{A}$ on $Y_{A}$ is defined. Let ||$_{A}$ denote the idele norm on the idele group $k_{A}^{\times}$; then, for $(x, s)$ in $Y_{A} \times \mathbf{C},|P f(x)|_{A}^{s}$ depends continuously on $x$ and holomorphically on $s$. We define the zeta function of $P f$ by the following integral :

$$
Z(s, \Phi)=\int_{Y_{A}} \Phi(x)|P f(x)|_{A}^{s}|\lambda \omega(x)|_{A},
$$

in which $\Phi$ is an arbitrary element of $\mathscr{S}\left(X_{A}\right)$. We shall first make the multiplicative calculation; this implies, in particular, that the above integral is absolutely convergent for $\operatorname{Re}(s)>2 n-1$.

We decompose $X_{A}$ into the product $X_{0} \times X_{\infty}$, in which $X_{0}$ is the restricted product of $X_{v}$ for all $v$ not in $S_{\infty}$ and $X_{\infty}$ the product of $X_{v}$ for all $v$ in $S_{\infty}$. Then, every element $\Phi$ of $\mathscr{S}\left(X_{A}\right)$ can be written as a linear combination of $\Phi_{0} \otimes \Phi_{\infty}$, in which $\Phi_{0}$ is in $\mathscr{S}\left(X_{0}\right)$ and $\Phi_{\infty}$ in $\mathscr{S}\left(X_{\infty}\right)$. We shall assume that $\Phi$ itself is of this form. Suppose that $v$ is nonarchimedian and that $\Phi_{v}$ is the characteristic function of $X_{v}^{0}$; then, by Lemma 4, we see that the corresponding local zeta function is given by

$$
\begin{aligned}
& \left(1-q^{-1}\right)^{-1} \int_{Y_{v}} \Phi_{v}(x)|P f(x)|_{v}^{s-(2 n-1)}|d x|_{v} \\
& =m\left(X_{v}^{0}\right) \prod_{i=1}^{n-1}\left(1-q^{-(2 i+1)}\right) \text {-times } \\
& \sum_{m=0}^{\infty} q^{-m(s-2 n+2)} \prod_{i=1}^{n-1}\left(1-q^{-2(i+m)}\right)\left(1-q^{-2 i}\right)^{-1},
\end{aligned}
$$

in which $q=q_{v}$. We observe that the above series converges absolutely to, say $S_{n}(s)$, for $\operatorname{Re}(s)>2 n-2$. On the other hand, if we put 


$$
F_{m, n}(t)=\prod_{i=1}^{n-1}\left(1-t^{i+m}\right)\left(1-t^{i}\right)^{-1}
$$

for $m \geqq 0, n \geqq 1$ with the understanding that $F_{m, 1}(t)=1$, we have $F_{m, n}(t)$ $=F_{m, n-1}(t)+t^{n-1} \boldsymbol{F}_{m-1, n}(t)$ for $m \geqq 1, n \geqq 2$; this implies that

$$
S_{n}(s)=q^{-s} S_{n}(s)+S_{n-1}(s-2)
$$

for $n \geqq 2$. Since $S_{1}(s)=\left(1-q^{-s}\right)^{-1}$, we get

$$
S_{n}(s)=\prod_{i=0}^{n-1}\left(1-q^{-(s-2 i)}\right)^{-1} ;
$$

hence the local zeta function becomes

$$
m\left(X_{v}^{0}\right) \prod_{i=1}^{n-1}\left(1-q^{-(2 i+1)}\right) \cdot \prod_{i=0}^{n-1}\left(1-q^{-(s-2 i)}\right)^{-1} .
$$

Also the archimedian part of the zeta function is given by the following Mellin-type transform:

$$
\int_{X_{\infty}} \Phi_{\infty}(x)|P f(x)|_{\infty}^{s-(2 n-1)}|d x|_{\infty}
$$

of $\Phi_{\infty}$, in which ||$_{\infty}$ and $|d x|_{\infty}$ denote the products of ||$_{v}$ and $|d x|_{0}$ for all $v$ in $S_{\infty}$. We observe that the above integral converges absolutely for $\operatorname{Re}(s)>2 n-1$. Therefore, if we denote by $\zeta_{k}(s)$ the Dedekind zeta function of $k$, the global zeta function $Z(s, \Phi)$ for $\Phi=\Phi_{0} \otimes \Phi_{\infty}$ coincides, except for an elementary factor, with the product of $\zeta_{k}(s) \zeta_{k}(s-2) \ldots$ $\zeta_{k}(s-2 n+2)$ and the above Mellin transform of $\Phi_{\infty}$ in the half plane defined by $\operatorname{Re}(s)>2 n-1$. In particular, since an arbitrary $\Phi$ is a linear combination of a finite number of special $\Phi$ 's of the form $\Phi_{0} \otimes \Phi_{\infty}$, the integral for $Z(s, \Phi)$ is absolutely convergent for $\operatorname{Re}(s)>2 n-1$.

We shall make the additive calculation vis-à-vis the above multiplicative calculation; this gives the analytic continuation and the functional equation of $Z(s, \Phi)$. For any $g^{*}$ in $G_{A}^{*}$, the Fourier transform of $\Psi(x)=\Phi\left(\rho\left(g^{*}\right) \cdot x\right)$ is given by

$$
\Psi^{*}(x)=\left|\operatorname{det}\left(g^{*}\right)\right|_{A}^{-(2 n-1)} \Phi^{*}\left({ }^{t} \rho\left(g^{*}\right)^{-1} \cdot x\right),
$$

and we have the Poisson formula:

$$
\sum_{\xi \in \dot{X}_{k}} \Psi(\xi)=\sum_{\xi \in X_{k}} \Psi^{*}(\xi) .
$$


Since we have $[x, y]=\frac{1}{2} \operatorname{tr}\left(x^{t} y\right)$, we get ${ }^{t} \rho(g)^{-1}=\rho\left({ }^{t} g^{-1}\right)$ for every $g$ in $G^{*}$; hence

$$
I\left(\Phi_{1}\right)=\int_{G_{A} / G_{k}}\left(\sum_{\xi \in X_{k}} \Phi_{1}\left(t \rho(g)^{-1} \cdot \xi\right)\right)|d g|_{A}
$$

for every $\Phi_{1}$ in $\mathscr{S}\left(X_{A}\right)$. After observing this, we replace $g^{*}$ by $u g$ with $u$ in $G_{A}^{*}$ and $g$ in $G_{A}$, and integrate both sides of the Poisson formula over $G_{A} / G_{k}$. Then, by using (3) and $\left|\omega_{r}(\rho(u) \cdot x)\right|_{A}=|\operatorname{det}(u)|_{A}^{2 r}\left|\omega_{r}(x)\right|_{A}$, we get

$$
\begin{aligned}
& \sum_{i \in k^{\star}} \int_{U(i) \Delta} \Phi(\rho(u) \cdot x)\left|\theta_{i}(x)\right|_{A}+\sum_{r=0}^{n-1} \sigma_{r}|\operatorname{det}(u)|_{A}^{-2 r} \\
& \quad=|\operatorname{det}(u)|_{A}^{-(2 n-1)}\left(\sum_{i \in k^{*}} \int_{U(i) A} \Phi^{*}\left(t \rho(u)^{-1} \cdot x\right)\left|\theta_{i}\right|_{A}+\sum_{r=0}^{n-1} \sigma_{r}^{*}|\operatorname{det}(u)|_{A}^{2 r}\right),
\end{aligned}
$$

in which

$$
\sigma_{r}=\int_{\left(U_{r}\right)_{A}} \Phi \cdot\left|\omega_{r}\right|_{A}, \quad \sigma_{r}^{*}=\int_{\left(U_{r}\right)_{A}} \Phi^{*} \cdot\left|\omega_{r}\right|_{A}
$$

for $0 \leqq r \leqq n-1$.

Let $d g^{*}$ denote a gauge form on $G^{*}$ defined over $k$. Since $Y$ satisfies the Witt condition relative to any field and $S p_{2 n}$ has 1 as its Tamagawa number, by the standard method we get

$$
\begin{aligned}
Z(s, \Phi) & =\int_{G_{A}^{*} / G_{k}^{*}}\left(\sum_{\xi \in Y_{k}} \Phi(\rho(u) \cdot \xi)\right)|\operatorname{det}(u)|_{A}^{s}|\lambda d u|_{A} \\
& =\int_{k_{A}^{\times} / k^{x}}|t|_{A}^{s}\left|\lambda t^{-1} d t\right|_{A} \int_{G_{A} / G_{k}}\left(\sum_{\xi \in \mathbf{Y}_{k}} \Phi(\rho(u g) \cdot \xi)\right)|d g|_{A},
\end{aligned}
$$

in which $t=\operatorname{det}(u)$. Therefore, if we put

$$
\Xi(t)=\sum_{i \in k^{\times}} \int_{U(i) \boldsymbol{A}} \Phi(\rho(u) \cdot x)\left|\theta_{i}(x)\right|_{A},
$$

we also have

$$
Z(s, \Phi)=\int_{k_{A^{\times} / k^{x}}} \Xi(t)|t|_{A}^{s}\left|\lambda t^{-1} d t\right|_{A}
$$

Let $f_{+}$denote the function on $\mathbf{R}$ defined by $f_{+}(\tau)=1$ for $\tau>1, f_{+}(\tau)=0$ for $\tau<1$, and $f_{+}(1)=\frac{1}{2}$; put $1=f_{+}+f_{-}$and

$$
Z_{ \pm}(s, \Phi)=\int_{k_{A}^{\times} / k \times} \Xi(t) f_{ \pm}\left(|t|_{A}\right)|t|_{A}^{s}\left|\lambda t^{-1} d t\right|_{A}
$$


then we have $Z(s, \Phi)=Z_{+}(s, \Phi)+Z_{-}(s, \Phi)$. Since the integral for $Z_{+}(s, \Phi)$ is absolutely convergent for $\operatorname{Re}(s)>2 n-1$ and since the absolute value of the integrand is a monotone increasing function of $\operatorname{Re}(s)$, it is absolutely convergent for every $s$; hence $Z_{+}(s, \Phi)$ is an entire function of $s$. After observing this, we multiply $f_{-}\left(|t|_{A}\right)|t|_{A}^{s}$ to $\left(3^{\prime}\right)$ and integrate both sides over $k_{A}^{\times} / k^{\times}$with respect to $\left|\lambda t^{-1} d t\right|_{A}$. Then we get

$$
\begin{aligned}
& Z_{-}(s, \Phi)+\rho_{k} \cdot\left(\sum_{r=0}^{n-1} \sigma_{r} \cdot(s-2 r)^{-1}\right) \\
& \quad=Z_{+}\left(2 n-1-s, \Phi^{*}\right)+\rho_{k} \cdot\left(\sum_{r=0}^{n-1} \sigma_{r}^{*} \cdot(s-2 n+1+2 r)^{-1}\right),
\end{aligned}
$$

in which $\rho_{k}$ is a positive constant which depends only on $k$; by taking $n=1$, we see that it is the residue at $s=1$ of $\zeta_{k}(s)$. We have thus obtained the following theorem:

THEOREM 2. The zeta function $Z(s, \Phi)$ of $P f$ has an analytic continuation to the entire s-plane as a meromorphic function with eventual poles of order 1 at $s=0,1, \cdots, 2 n-1$; it satisfies the functional equation

$$
Z(s, \Phi)=Z\left(2 n-1-s, \Phi^{*}\right)
$$

and, if $\Phi$ is of the form $\Phi_{0} \otimes \Phi_{\infty}$ and $\operatorname{Re}(s)>2 n-1$, we have

$$
\begin{aligned}
Z(s, \Phi)= & \text { elementary factor } \cdot \prod_{i=0}^{n-1} \zeta_{k}(s-2 i) \\
& \cdot \int_{X_{\infty}} \Phi_{\infty}(x)|P f(x)|_{\infty}^{s-(2 n-1)}|d x|_{\infty} .
\end{aligned}
$$

We can make the multiplicative calculation also for an archimedian $k_{v}$ provided that we take a special $\Phi_{v}$. For instance, if $e$ denotes the sum of $e_{1}, a$ an upper triangular matrix with $a_{2}=a_{4}=\cdots=1$, and $\Phi_{v}\left({ }^{t} a e a\right)$ $=\exp \left(-\operatorname{tr}\left({ }^{t} a \bar{a}\right)\right)$, then, by a calculation similar to Siegel [6], I, pp. 384385 , we get

$$
\int_{X_{v}} \Phi_{v}(x)|P f(x)|_{v}^{s-(2 n-1)}|d x|_{v}=\text { const. } \prod_{i=0}^{n-1} \Gamma\left(r_{v}(s-2 i)\right),
$$

in which $\Gamma$ denotes the gamma function and $r_{v}=\frac{1}{2}\left[k_{v}: \mathbf{R}\right]$, i.e., $r_{v}=\frac{1}{2}$ for $k_{v}=\mathbf{R}$ and $r_{v}=1$ for $k_{v}=\mathbf{C}$. 


\section{Appendix}

We shall first show that our treatment of the split case can be carried over to the non-split case by an additional observation on Galois cohomology: let $\boldsymbol{k}$ denote a quaternion algebra defined over a field $k$; consider the vector space $H_{n}(\boldsymbol{k})$ of hermitian elements in $M_{n}(\boldsymbol{k})$ relative to the usual involution $\xi \rightarrow \xi^{\prime}$ of $\boldsymbol{k}$; then $G=S L_{n}(\boldsymbol{k})$ acts on $X=H_{n}(k)$ as $\rho(g) \cdot x=g x^{t} g^{\prime}$. In this way, we get a rational representation $\rho$ of $G$ in $X$ defined over $k$. If $k$ splits over $k, \rho$ is equivalent over $k$ to the representation of $S L_{2 n}$ in the vector space of alternating matrices of degree $2 n$; hence, if $\boldsymbol{k}$ does not split over $k$, we get a $k$-form of this representation. (If $k$ is an algebraic number field, there is no other $k$-form.) The ring of $G$-invariants on $X$ is generated by a homogeneous polynomial $f$ of degree $n$ with coefficients in $k$ normalized by $f\left(1_{n}\right)=1$; $f$ is the norm form of $X$ considered as a simple Jordan algebra defined over $k$.

Suppose that $k$ is an algebraic number field and $U$ any $G$-orbit in $X$ defined over $k$. Then (i) two points of $U_{k}$ are $G_{k}$-equivalent if and only if they are $G_{A}$-equivalent; (ii) every $G_{A}$-orbit in $U_{A}$ contains a $k$ rational point; (iii) the number of $G_{A}$-orbits in $U_{A}$ is finite. These follow from some well-known properties of quaternionic hermitian forms. On the other hand, the Tamagawa numbers of $G$ and its stabilizer at any point of $U_{k}$ are known to be 1 [cf. 7,9]. Therefore, if the Poisson formula can be applied to the adelized space of invariants, we will get the Siegel formula. The only point which we have to make certain is the validity of the asymptotic estimates at those finite number of $v$ 's where $f$ is not equivalent over $k_{v}$ to $P f$.

Let $x$ denote a point of $X=H_{n}(\boldsymbol{k})$ and write $x$ as

$$
x=\left[\begin{array}{ll}
y & z \\
t^{\prime} & \alpha
\end{array}\right],
$$

in which $y$ is in $Y=H_{n-1}(\boldsymbol{k})$; let $f(y)$ denote the norm of $y$; then we have

$$
f(x)=\alpha \cdot f(y)+Q_{y}(z) .
$$

We observe that the $z$-space $Z$ is a vector space of dimension $4(\dot{n}-1)$ defined over $k$ and $Q_{y}$ is a quadratic form on $Z$ also defined over $k$ with $f(y)^{4(n-2)}$ as its discriminant. This follows from Lemma 1 . Therefore 
the proof of Lemma 9 carries over to the present case by just replacing $P f$ by $f$. (If we were interested only in $P f$, we could have used

$$
P f(x)=\sum_{i=1}^{2 n-1}(-1)^{i-1} P f\left(x_{i}\right) x_{i, 2 n}
$$

instead of $P f(x)=\alpha \cdot P f(y)+Q_{y}(z)$ and thus avoided the use of $\left(1^{\prime}\right)$.) In this way, we get Theorem 1 for $f$ instead of $P f$.

As for Theorem 2, put $G^{*}=G L_{n}(k)$ and $Y=X-f^{-1}(0)$. Then (i) two points of $Y_{k}$ are $G_{k}^{*}$-equivalent if and only if they are $G_{A}^{*}$-equivalent; (ii) every $G_{A}^{*}$-orbit in $Y_{A}$ contains a $k$-rational point; (iii) the number of $G_{A}^{*}$-orbits in $Y_{A}$ is finite. Since we have $H^{1}\left(K, G^{*}\right)=1$ for every $K$, the verification of these is simpler in the present case than in the previous case. At any rate, once we have those, by a similar argument as in the split case, we get Theorem 2 for $f$ instead of $P f$.

Finally, we shall formulate a problem and give its motivation; as above, let $f$ denote the norm form of the simple Jordan algebra $X=$ $H_{n}(\boldsymbol{k})$, in which $\boldsymbol{k}$ is any quaternion algebra defined over an algebraic number field $k$. We put $[x, y]=\frac{1}{2} \operatorname{tr}(x y+y x)$ and define the Fourier transformation $\mathbf{r}$ as

$$
(\mathbf{r} \Phi)(x)=\int_{X_{A}} \Phi(y) \chi([x, y])|d y|_{A}
$$

for every $\Phi$ in $\mathscr{S}\left(X_{A}\right)$; r extends uniquely to a unitary operator, also denoted by $\mathbf{r}$, of $L^{2}\left(X_{A}\right)$. We take $i^{*}$ from $k_{A}$ and consider the unitary operator $\mathbf{t}\left(i^{*}\right)$ defined by

$$
\left(\mathbf{t}\left(i^{*}\right) \Phi\right)(x)=\Phi(x) \chi\left(f(x) i^{*}\right)
$$

for every $\Phi$ in $L^{2}\left(X_{A}\right)$. Let $M(f)_{A}$ resp. $M(f)_{k}$ denote the subgroups of the unitary group Aut $\left(L^{2}\left(X_{A}\right)\right)$ generated by $\mathbf{r}$ and $\mathbf{t}\left(i^{*}\right)$ for all $i^{*}$ in $k_{A}$ resp. in $k$. The problem is to determine the structure of $M(f)_{k}$ and also of $M(f)_{A}$. We observe that $I$ is invariant under r, i.e., $I(\mathbf{r} \Phi)=I(\Phi)$ for every $\Phi$ in $\mathscr{S}\left(X_{A}\right)$; that $E$ is invariant under $\mathbf{t}\left(i^{*}\right)$ for all $i^{*}$ in $k$. Therefore $I=E$ is invariant under the group $M(f)_{k}$. If $n=1, M(f)_{k}$ is a semidirect product of $k^{2}$ by the cyclic group of order 4 consisting of powers of $\mathbf{r}$; if $n=2, M(f)_{k}$ is an extension of $S L_{2}(k)$ by a cyclic group of order 2. In both cases, the invariance of $I=E$ under $M(f)_{k}$ forms a basis of the fact that the Siegel formula gives rise to functions on $M(f)_{A}$ which are "automorphic" under its lattice $M(f)_{k}$. If we disregard 
finite groups, $M(f)_{A}$ is the "Heisenberg group" for $n=1$ and the "metaplectic group" of Weil for $n=2$. Nothing of this kind seems to be known for $n \geqq 3$.

\section{REFERENCES}

[1] C. Chevalley, The construction and study of certain important algebras, Pub. Math. Soc. Japan, 1 (1955).

[ 2 ] J. Igusa, Some observations on the Siegel formula, Rice Univ. Studies, 56 (1970), 67-75.

[3] J. Igusa, On certain representations of semi-simple algebraic groups and the arithmetic of the corresponding invariants (1), Inventiones Math., 12 (1971), 62-94.

[ 4 ] S. Lang, Diophantine geometry, Interscience Pub., 11 (1962).

[5] J. G. M. Mars, Les nombres de Tamagawa de certains groupes exceptionels, Bull. Soc. Math. France, 94 (1966), 97-140.

[ 6 ] C. L. Siegel, Gesammelte Abhandlungen I-III, Springer (1966).

[ 7 ] A. Weil, Adels and algebraic groups, Lecture Note, Princeton (1961).

[8] A. Weil, Sur certain groupes d'opérateurs unitaires, Acta Math., 111 (1964), 143-211.

[ 9 ] A. Weil, Sur la formule de Siegel dans la théorie des groupes classiques, Acta Math., 113 (1965), 1-87.

The Johns Hopkins University

Baltimore, Maryland 21218 